\title{
Comparison of five commercial kits for the determination of glycosylated haemoglobin
}

\author{
D NORCLIFFE, EM TURNER \\ From the Haematology Department, Northern General Hospital, Sheffield
}

SUMMARY Five commercial chromatography kits for the measurement of glycosylated haemoglobin have been compared using blood specimens obtained from diabetic patients and from non-diabetic controls. Assessment was based on accuracy, general impressions of practicality, and overall performance. The precision of each technique was assessed by selecting five diabetic and five non-diabetic specimens and testing each one by each technique. All systems tested were of a high standard and the advantages and disadvantages of each system are discussed.

Glycosylated haemoglobin $\left(\mathrm{HbA}_{1}\right)$ is a minor component of normal adult haemoglobin ( $\mathrm{HbA})$, and in healthy subjects constitutes about $7 \%$ of the total haemoglobin. It consists of several sub-fractions ${ }^{1-3}$ : $\mathrm{HbA}_{1 \mathrm{a}}, \mathrm{HbA}_{1 \mathrm{~b}}$, and $\mathrm{HbA}_{1 \mathrm{c}} . \mathrm{HbA}_{1 \mathrm{c}}$ is the major subfraction and accounts for roughly $80 \%$ of glycosylated haemoglobin. All three sub-fractions are increased proportionately in patients with diabetes mellitus, ${ }^{4}$ and the total amount of $\mathrm{HbA}_{1}$ is thought to be a good indicator of long term metabolic control.

Most commercial kit methods for measurement of $\mathrm{HbA}_{1}$ are chromatographic and, compared with other methods, these have the disadvantage of being temperature dependent. Furthermore, not all commercial kits give the user the option of eliminating aldimine, which is the unstable intermediate of the glycosylation reaction. Since the aldimine fraction normally accounts for less than $10 \%$ of $\mathrm{HbA}_{1 \mathrm{c}}$ it has been suggested that the elimination is generally unnecessary unless an acute change in blood glucose concentration has recently occurred ${ }^{5}$ or the patient is uraemic or has a history of alcoholism.

\section{Material and methods}

Five commercial chromatography kits for the measurement of glycosylated haemoglobin were assessed and compared. The kits used were $\mathrm{HbA}_{1}$ Test Combination (BCL, Boehringer Corporation), Bio Rad (Bio Rad Laboratories Ltd), DCA Stat Glycos (IRE Ltd), Isolab Quick-Sep (Advanced Laboratory Techniques), and Sigma (Sigma Corporation). All were 100 test size, though some of the manufacturers produced 20 test sizes. Assessment was based on several factors: accuracy and precision, general impressions of practicality, and overall performance.

Blood specimens were obtained from diabetic patients at various levels of control and from known non-diabetic subjects. $\mathrm{HbA}_{1}$ concentration was determined within the stipulated time period of each kit-that is, all samples tested were less than one week old and had been stored at $4^{\circ} \mathrm{C}$. Edetic acid anticoagulated blood was used throughout. Each batch of tests was run simultaneously under the same temperature conditions and using the same specimens.

Non-diabetic subjects were chosen from laboratory staff and hospital inpatients with various disorders; care was taken so as not to select those who were known diabetics. All results are summarised in Tables 1-4.

A precision check of each technique was carried out by selecting specimens from five diabetic and five non-diabetic patients. Each specimen was tested by each technique. Thus each technique was subjected to a precision check by allowing each sample to be assayed five times simultaneously under identical conditions. The results are shown in Table 2 .

Table 1 Results of tests on controls

\begin{tabular}{lllll}
\hline Kit & $\begin{array}{l}\text { No of } \\
\text { samples }\end{array}$ & $\begin{array}{l}\text { Mean } \\
H b A_{1} \%\end{array}$ & $\begin{array}{l}\text { Standard } \\
\text { deviation }\end{array}$ & $\begin{array}{l}\text { Range } \\
\text { Mean } \pm 2 S D\end{array}$ \\
\hline BCL & 51 & $5 \cdot 3$ & $1 \cdot 08$ & $3 \cdot 1-7 \cdot 5$ \\
Bio Rad & 51 & $5 \cdot 85$ & $1 \cdot 40$ & $3 \cdot 0-8 \cdot 7$ \\
DCA & 51 & $5 \cdot 9$ & $1 \cdot 18$ & $3 \cdot 6-8 \cdot 8$ \\
Isolab & 51 & $5 \cdot 3$ & $1 \cdot 10$ & $3 \cdot 1-7 \cdot 5$ \\
Sigma & 51 & $5 \cdot 7$ & $1 \cdot 62$ & $2 \cdot 4-8 \cdot 9$ \\
\hline
\end{tabular}


Table 2 Results of precision check

\begin{tabular}{|c|c|c|c|c|c|c|c|c|}
\hline \multirow[t]{2}{*}{ Kit } & \multicolumn{2}{|c|}{ No of sainples } & \multicolumn{2}{|c|}{ Mean $H b A_{1} \%$} & \multicolumn{2}{|c|}{$\begin{array}{l}\text { Standard } \\
\text { deviation }\end{array}$} & \multicolumn{2}{|c|}{$\begin{array}{l}\text { Coefficient of } \\
\text { variation }\end{array}$} \\
\hline & $N D$ & $D$ & $N D$ & $D$ & $N D$ & $\cdot D$ & $N D$ & $D$ \\
\hline $\begin{array}{l}\text { BCL } \\
\text { Bio Rad } \\
\text { DCA } \\
\text { Isolab } \\
\text { Sigma }\end{array}$ & $\begin{array}{l}10 \\
10 \\
10 \\
10 \\
10\end{array}$ & $\begin{array}{l}10 \\
10 \\
10 \\
10 \\
10\end{array}$ & $\begin{array}{l}5 \cdot 1 \\
5 \cdot 1 \\
4 \cdot 8 \\
3 \cdot 7 \\
4 \cdot 8\end{array}$ & $\begin{array}{l}13 \cdot 1 \\
10 \cdot 3 \\
12 \cdot 8 \\
10 \cdot 6 \\
18 \cdot 2\end{array}$ & $\begin{array}{l}0 \cdot 10 \\
0.39 \\
0.33 \\
0 \cdot 24 \\
0.46\end{array}$ & $\begin{array}{l}0.24 \\
0.29 \\
1.00 \\
0.43 \\
1.31\end{array}$ & $\begin{array}{l}1 \cdot 94 \\
7 \cdot 60 \\
6 \cdot 80 \\
6 \cdot 43 \\
9 \cdot 50\end{array}$ & $\begin{array}{l}1 \cdot 80 \\
2 \cdot 80 \\
7 \cdot 85 \\
4 \cdot 00 \\
7 \cdot 18\end{array}$ \\
\hline
\end{tabular}

$\mathrm{ND}=$ non-diabetic, $\mathrm{D}=$ diabetic.

\section{METHODS}

\section{BCL HbA Test Combination.}

The BCL ( $\mathrm{HbA}_{1}$ Test Combination) kit consists of 100 micro disposable coltumns prefilled with resin, a bottle each of lysing agent, first elution buffer, second elution buffer, and an implement for releasing the seal on each column.

Fifty microlitres of whole blood is added to $200 \mu \mathrm{l}$ of lysing agent, mixed, and allowed to stand for 10 min. During this time the temperature of the environment is noted.

The excess buffer is drained through the column, which contains a filter disc to stop it drying out, and $50 \mu$ l of haemolysate is added to the top of the column bed. Penetration of the lysate into the column rapidly occurs, and $200 \mu \mathrm{l}$ of the first elution buffer is then added. Once this has penetrated the column a collection tube is placed underneath and $4 \mathrm{ml}$ of the first elution buffer is added in order to elute the glycosylated fraction. Collection of this fraction is complete in about $15 \mathrm{~min}$, after which the collection tube is changed and $4 \mathrm{ml}$ of the second elution buffer is added to the column, which elutes the remainder of the haemolysate.

Once complete the second fraction is diluted with distilled water and the absorbance of each fraction is measured in a spectrophotometer at a wavelength of $415 \mathrm{~nm}$. The glycosylated fraction is then calculated as a percentage of the total after making a correction for temperature using the chart provided. If a $\mathrm{BCL}$ rack is used in conjunction with a water bath at $23^{\circ} \mathrm{C}$, however, conversion to the appropriate temperature is not required.

\section{Bio Rad kit}

The Bio Rad kit contains 100 prefilled disposable columns and two one litre bottles of elution/ developing reagent, together with a bottle of lysing agent.

One hundred microlitres of whole blood is added to $500 \mu \mathrm{l}$ of lysing agent, mixed, and allowed to stand. The columns are prepared by shaking, in order to resuspend the resin, and releasing the seals on the top and bottom. The excess buffer is then allowed to drain from the columns.

One hundred microlitres of the lysate is added directly on to the surface of the resin bed and allowed to penetrate. A collection tube is placed underneath and $10 \mathrm{ml}$ of elution/developing reagent is added. While the glycosylated fraction is eluting a "total" dilution of the lysate is made by adding 100 $\mu \mathrm{l}$ to $10 \mathrm{ml}$ of elution/developing reagent.

The elution process is complete in about $45 \mathrm{~min}$ and the absorbance of both fractions is read in a spectrophotometer at a wavelength of $415 \mathrm{~nm}$. The percentage of glycosylated haemoglobin is thus calculated and no correction is made for temperature. A temperature controlled rack is available from Bio Rad.

\section{DCA Stat Glycos kit}

The DCA Stat Glycos kit is a unique system consisting of a single bottle of resin and 100 tubes and separators, which are reusable. A bottle of lysing agent sufficient for 100 tests and a lyophilised specimen of a standard and control are also included. Once reconstituted the standard and control will store in aliquots of $100 \mu \mathrm{l}$ for two months at $-20^{\circ} \mathrm{C}$. The value of the standard and the range of the control are printed on each vial.

One hundred microlitres of whole blood is added to $500 \mu \mathrm{l}$ of lysing agent and allowed to stand. Three millilitres of well mixed resin is pipetted into the tubes provided and $100 \mu$ l of the lysate is added. The filter separator is then placed in each tube and mixed continually for $10 \mathrm{~min}$.

After mixing, the glycosylated fraction-that is, the fraction not bound to the resin-is eluted by pushing the filter separator towards the bottom of the tube. The eluate may then be poured directly from the separator into the spectrophotometer for the absorbance reading at $415 \mathrm{~nm}$ wavelength. The absorbance of the "total" fraction made by adding $20 \mu \mathrm{l}$ of lysate to $5 \mathrm{ml}$ of distilled water is also measured and the percentage of glycosylated haemoglobin is calculated. 
The calculated value of the standard and control is compared with the stated values and the correction factor obtained. This correction factor is then applied to the test results, thus eliminating the need for temperature correction.

\section{Isolab kit}

The Isolab kit consists of 100 prefilled disposable columns, first and second elution reagents, and a bottle of lysing agent. Also included is a bottle of reagent which, when added to the lysate, will eliminate the aldimine fraction. Hence the user is provided with a choice of whether to eliminate the aldimine or not. In this trial the eliminator was not used.

Fifty microlitres of whole blood is added to $200 \mu \mathrm{l}$ of lysing agent and allowed to stand. The excess buffer is drained through the column and the top of the filter disc, which prevents drying of the column, is positioned on the surface of the bed. Fifty microlitres of lysate is added to the column and once penetration has occurred a further $200 \mu$ l of the first elution buffer is added and allowed to drain. A collection tube is then placed underneath and $4 \mathrm{ml}$ of the first elution buffer is added to the column. The eluate collected is thus the glycosylated fraction and this step is complete in about $20 \mathrm{~min}$. The collection tube is then changed and $4 \mathrm{ml}$ of the second solution is added. This releases the remaining haemoglobins from the column, which are then diluted with distilled water. The absorbance of both fractions is read on a spectrophotometer at a wavelength of $415 \mathrm{~nm}$ and the percentage of glycosylated haemoglobin is calculated. This is then amended by adjusting the results to correspond with the environmental temperature.

Table 3 Results of tests on diabetic patients

\begin{tabular}{lllll}
\hline Kit & $\begin{array}{l}\text { No of } \\
\text { samples }\end{array}$ & $\begin{array}{l}\text { Mean } \\
\text { HbA, } \%\end{array}$ & $\begin{array}{l}\text { Standard } \\
\text { deviation }\end{array}$ & $\begin{array}{l}\text { Range } \\
\text { Mean } \pm 2 S D\end{array}$ \\
\hline BCL & 90 & $10 \cdot 05$ & $2 \cdot 16$ & $5 \cdot 8-14 \cdot 1$ \\
Bio Rad & 90 & $9 \cdot 4$ & $1 \cdot 97$ & $5 \cdot 4-13 \cdot 3$ \\
DCA & 90 & $7 \cdot 5$ & $1 \cdot 76$ & $4 \cdot 0-11 \cdot 0$ \\
Isolab & 90 & $9 \cdot 5$ & $2 \cdot 04$ & $5 \cdot 4-13 \cdot 6$ \\
Sigma & 90 & $9 \cdot 3$ & $2 \cdot 26$ & $4 \cdot 8-13 \cdot 8$ \\
\hline
\end{tabular}

Table 4 Differentiation between non-diabetic and diabetic values

\begin{tabular}{ll}
\hline Kit & $\begin{array}{l}\text { \% Overlap of } \mathrm{HbA}_{1} \text { in diabetic and } \\
\text { non-diabetic values }\end{array}$ \\
\hline BCL & $15 \cdot 0$ \\
Bio Rad & $32 \cdot 0$ \\
DCA & $64 \cdot 8$ \\
Isolab & $20 \cdot 0$ \\
Sigma & 35.9 \\
\hline
\end{tabular}

\section{Sigma kit}

The Sigma kit consists of 100 prefilled disposable columns, a single bottle of buffer, and a bottle of lysing agent. The columns may, if required, be prepared by shaking to resuspend the resin or by simply draining off the excess buffer through the column, though the latter method may prolong the technique by a few minutes.

One hundred microlitres of whole blood is added to $500 \mu \mathrm{l}$ of lysing agent and allowed to stand after mixing. Fifty microlitres of this lysate is then added directly on to the resin bed surface and allowed to enter. A collection tube is then placed underneath the column and $5 \mathrm{ml}$ of the buffer solution carefully added so as not to disturb the resin, and the eluate is collected. The process is complete in about $15 \mathrm{~min}$, thus allowing time for a dilution of the total haemoglobin to be made by adding $20 \mu \mathrm{l}$ of lysate to $10 \mathrm{ml}$ of the buffer.

The absorbance of both fractions is read in a spectrophotometer at a wavelength of $415 \mathrm{~nm}$ and the percentage of glycosylated haemoglobin is calculated. A correction factor is used in calculating the final result in order to compensate for environmental temperature.

\section{General impressions}

The BCL kit is easy to use and the inclusion of a simple implement for releasing the seals on the columns is particularly useful. A filter disc prevents the column from drying out and keeps the resin compact when adding the reagents, one of the similarities to the Isolab technique. The temperature correction chart, however, accounts for temperatures no less than $20^{\circ} \mathrm{C}$, which during certain times of the year may be difficult to maintain. This may be overcome by use of the BCL temperature controlled rack.

The Bio Rad kit has the distinction of being the first commercial assay kit for glycosylated haemoglobin on the UK market. It contains a comprehensive instruction booklet, which includes a short history of the present technique, together with many references and the normal ranges produced by many workers. Unfortunately, however, it does not contain facilities for correction due to temperature differences, but recommends that the test be carried out at $22^{\circ} \mathrm{C}$. Despite this, the kit is easy to use and a reliable result may be obtained within $1 \mathrm{~h}$. From the point of view of comparison it is unfortunate that the laboratory was not aware of the existence of the Bio Rad temperature controlling rack. It should be noted that the trial involved the Bio Rad HbA, kit and not the Bio Rad $A_{1 c}$ specific kit, which is now marketed.

The DCA kit is the cheapest of those tested and 


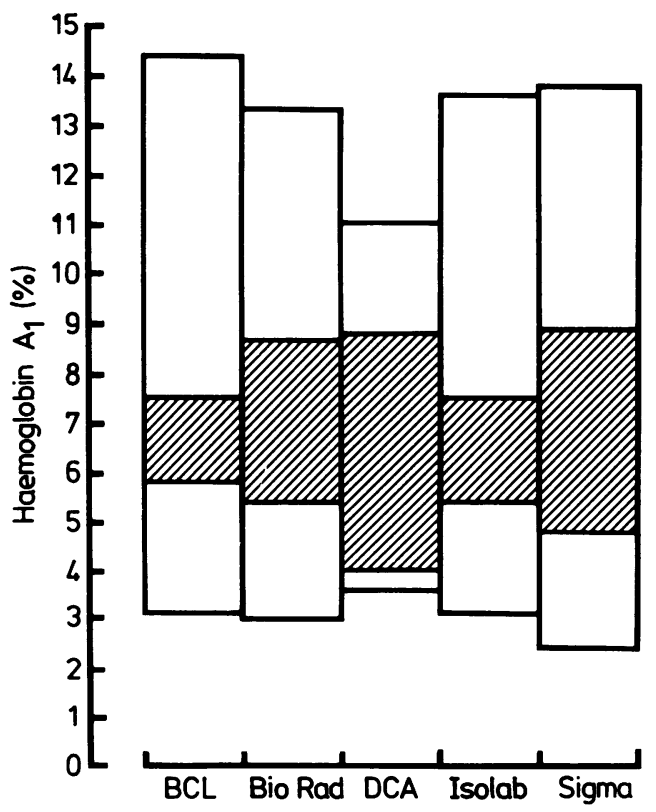

Normal and diabetic ranges of each technique. Shaded areas represent overlap between the two ranges.

the cost may be reduced even further after the initial purchase of the first kit as the tubes and filter separators are reusable. The kit is easy to use and is the only technique of those studied which does not involve a sub-column collection. A result may be obtained within $30 \mathrm{~min}$, and since the kit contains standard and control no correction for temperature per se is required, but an overall correction factor is applied.

The Isolab system is remarkably similar to that of BCL in appearance. It contains a filter disc on the surface of the resin bed to prevent drying out and to aid the addition of buffers. The temperature correction chart provided covers a range from $18-28^{\circ} \mathrm{C}$ and hence is more adaptable to a particular environment than BCL. It also contains an aldimine eliminator to be added, if required, at the users discretion.

The Sigma technique is quick, producing a result within $45 \mathrm{~min}$. The kit is easy to use and the temper- ature correction factor makes the calculation of results relatively easy and eliminates the use of a correction chart.

\section{Discussion}

All systems tested were of a high standard and difficulty in choosing any particular one inevitably occurred. The choice will vary between establishments depending on the anticipated number and the budget available.

The BCL system showed excellent precision with coefficients of variation of $1.8 \%$ on a specimen with a $\mathrm{HbA}_{1}$ concentration in the diabetic range and $1.9 \%$ in the normal range. The Figure shows BCL to have the smallest overlap area of those tested. It was $15.0 \%$ of the total ranges, and hence shows more differentiation between the two ranges than any of the other techniques.

The Bio Rad system compares remarkably well despite the fact that temperature correction facilities were not used. An overlap of ranges of $32 \%$ is shown in the Figure.

The DCA system is inexpensive and easy to use but unfortunately performed rather disappointingly on the precision check. It also had an overlap of ranges of $64.8 \%$ (Figure). It is the only system of those tested which includes standards and controls 0

The Isolab system shows mid-range precision per formance. The inclusion of the filter disc and the wide temperature range in which it can be used make it versatile. The Figure shows a $20 \%$ overlap of ranges, which compares well with its competitors.

The Sigma system performed disappointingly, with a coefficient of variation $9.5 \%$ in the normal range and an overlap of ranges of $35.9 \%$. Despite the ease of use and speed, it would probably not be chosen as the ideal system.

The sum of the values obtained for coefficient of variation in both ranges and the percentage overlap of ranges is expressed as the "summary index" and is shown in Table 5.

We thank the following for their help and consideration: friends and colleagues at the Northern General Hospital, Sheffield, particularly Dr MJ Brown, Dr

Table 5 Summary of results

\begin{tabular}{lllll}
\hline & \multicolumn{2}{l}{ Coefficient of variation (\%) } & $\begin{array}{c}\text { \% Overlap of } \\
\text { ranges }\end{array}$ & "Summary index" \\
\cline { 2 - 5 } & Non-diabetic & Diabetic & $15 \cdot 0$ & $18 \cdot 74$ \\
\hline BCL & $1 \cdot 94$ & $1 \cdot 80$ & $20 \cdot 0$ & $30 \cdot 43$ \\
Isolab & $6 \cdot 43$ & $4 \cdot 00$ & $32 \cdot 0$ & $42 \cdot 40$ \\
Bio Rad & $7 \cdot 60$ & $2 \cdot 80$ & $35 \cdot 9$ & $52 \cdot 58$ \\
Sigma & $9 \cdot 50$ & $7 \cdot 18$ & $64 \cdot 8$ & $79 \cdot 45$ \\
DCA & $6 \cdot 80$ & $7 \cdot 85$ & \\
\hline
\end{tabular}


ACK Lawrence, Mr JM Bevington, and Mrs J Woolley, who typed this report. Our gratitude is also extended to the following representatives: $\mathrm{Mr} \mathrm{K}$ Aston (Bio Rad), Mr J Coward (IRE), and Mr C Sloane (BCL).

\section{References}

' Allen DW, Schroeder WA, Balog J. Observations of the chromatographic heterogeneity of normal adult and fetal human haemoglobin: a study of the effects of crystallisation and chromatography on the heterogeneity and iso leucine content. J Am Chem Soc 1958;80:1628-34.
${ }^{2}$ Clegg AG, Schroeder WA. A chromatographic study of the minor components of normal adult haemoglobin including a comparison of haemoglobin from normal and phenylketonuric individuals. J Am Chem Soc 1959;81:6065-9.

${ }^{3}$ Shneck AG, Schroeder WA. The relation between the minor components of whole normal adult haemoglobin as isolated by chromatography and stack block electrophoresis. J Am Chem Soc 1961;83:1472.

${ }^{4}$ Huisman TH, Dozy AM. Studies on the heterogeneity of haemoglobin $\mathbf{v}$ binding of haemoglobin with oxidised glutathione. J Clin Lab Med 1962;60:302-19.

5 Costa J. Effects of acute changes in blood glucose on $\mathrm{HbA}_{1}$. Diabetes 1980;29:623-9.

Requests for reprints to: Mr D Norcliffe, Northern General Hospital, Herries Road, Sheffield S5 7AU, England. 